\title{
Comparison of the prognostic value of longitudinal measurements of systemic inflammation in patients undergoing curative resection of colorectal cancer
}

\author{
G J K Guthrie *, , C S D Roxburgh ${ }^{1}$, O M Farhan-Alanie ${ }^{1}$, P G Horgan ${ }^{1}$ and D C McMillan ${ }^{1}$ \\ ${ }^{1}$ Academic Unit of Surgery, School of Medicine-University of Glasgow, Royal Infirmary, Glasgow G31 2ER, UK
}

Background: The systemic inflammation-based prognostic scores, modified Glasgow Prognostic Score (mGPS) and the neutrophil-lymphocyte ratio (NLR) are now recognised to be useful in predicting survival in a variety of solid organ malignancies, including colorectal cancer (CRC) before treatment. However, there would appear to have been no direct comparison of these longitudinal measurements of systemic inflammation. Therefore, the aim of the present study was to compare the prognostic value of longitudinal measures of systemic inflammation, the MGPS and NLR in patients undergoing potentially curative resection for CRC.

Methods: Three hundred and twenty-six patients underwent potentially curative resection for CRC between 2006 and 2010. Full biochemical and haematological data both pre- and post-operatively (3-6 months) were available for 206 patients.

Results: In 206 patients, there was no significant overall change in either the mGPS or the NLR, from pre- to post-operatively. On univariate survival analysis, T-stage $(P<0.001)$, tumour, node, metastasis stage $(P<0.005)$, pre-operative mGPS $(P<0.05)$, preoperative NLR $(<0.05)$, post-operative mGPS $(P<0.001)$ and post-operative NLR $(P<0.005)$ were associated with cancer-specific survival. On multivariate survival analysis, comparing pre-operative mGPS and NLR, both pre-operative mGPS and NLR were independently associated with reduced cancer-specific survival (mGPS hazard ratio (HR) 1.97, Cl 1.16-3.34, $P<0.05$, and NLR HR $3.07, \mathrm{Cl} 1.23-7.63, P<0.05)$. When the same multivariate comparison was carried out on post-operative data, only the postoperative mGPS was independently associated with cancer-specific survival (HR 4.81, Cl 2.13-10.83, $P<0.001$ ).

Conclusion: The results of the present study support the longitudinal assessment of the systemic inflammatory response, in particular the mGPS, in patients undergoing potentially curative resection for CRC.

Colorectal cancer (CRC) is the third most common cancer in males and females in the United Kingdom with $\sim 40000$ new cases recorded each year, and is the second most common cause of cancer death in both men and women in the United Kingdom with 16000 deaths per year (Cancerstats, 2010). Despite improvements in treatment, outcomes remain poor with approximately half of those undergoing curative surgery subsequently dying from the disease (Oliphant et al, 2013).
Cancer-associated inflammation, both in the tumour microenvironment and in the systemic circulation, is now widely recognised to be a key determinant of disease progression and survival in CRC. In particular, many studies have shown that the host response in the form of systemic inflammation is a key factor in determining outcomes in CRC, and as such the measurement of various circulating markers of systemic inflammation are useful in predicting survival. Indeed, it has been shown that a simple 
objective scoring system, that is, the modified Glasgow Prognostic Score (mGPS), which measures systemic inflammation using C-reactive protein (CRP) and albumin, is effective at predicting overall and cancer-specific survival in a variety of solid organ malignancies including CRC (McMillan, 2012). Similarly, it has become clear that the systemic inflammatory response results in changes in circulating white blood cells, and that components of the full blood count, in particular the neutrophil-lymphocyte ratio (NLR), also are useful in predicting overall and cancer-specific survival in a variety of solid organ malignancies, including CRC (Guthrie et al, 2013).

Recently, these systemic inflammation-based prognostic scores, mGPS and NLR were compared in a large cross-sectional cohort of unselected patients with cancer (Proctor et al, 2011); however, to our knowledge, there has been no direct comparison of the longitudinal measurement of the mGPS and NLR.

The clinical utility of such systemic inflammation-based prognostic scores would be enhanced if they were shown to have prognostic value over time and after therapeutic intervention. This would provide the basis of interventions directed at systemic inflammation, maintaining low levels of systemic inflammation and active surveillance, following resection of the CRC.

Therefore, the aim of the present study was to compare the prognostic value of longitudinal measurements of systemic inflammation in patients undergoing curative resection of the CRC.

\section{MATERIALS AND METHODS}

Patients with histologically proven CRC who, on the basis of laparotomy findings and pre-operative abdominal computed tomography, were considered to have undergone potentially curative resection between 2006 and 2010 in a single surgical unit at Glasgow Royal Infirmary were included in the study. The patients were identified from a prospectively maintained database and included both elective and emergency resections. Exclusion criteria were: clinical evidence of active infection, presence of a chronic inflammatory condition, and death within 30 days of surgery. Tumours were staged using the conventional tumour, node, metastasis (TNM) staging system, 7th Edition, 2010 (AJCC, 2010).

Longitudinal measurements of a differential white cell count, neutrophil and lymphocyte counts, CRP and albumin were recorded before surgery and between 3 and 6 months (median 3.5 months) following surgery.

The mGPS was constructed using CRP and albumin using previously described thresholds (McMillan, 2012). A score of 2 was allocated to those patients with both elevated CRP $\left(>10 \mathrm{mgl}^{-1}\right)$ and hypoalbuminaemia $\left(<35 \mathrm{~g}^{-1}\right)$, those with only an elevated CRP $\left(>10 \mathrm{mgl}^{-1}\right)$ were allocated a score of 1 , whereas those with normal CRP $\left(\leqslant 10 \mathrm{mgl}^{-1}\right)$ were allocated a score of 0 .

The NLR ratio was constructed using previously documented thresholds (Guthrie et al, 2013). Briefly, NLR was determined by dividing the absolute neutrophil count by the absolute lymphocyte count; the NLR data was then dichotomised using the most commonly used threshold of $\leqslant 5: 1$ out of $\geqslant 5: 1$, and the NLR given a score of $0(\leqslant 5: 1=$ low $)$ and $1(\geqslant 5: 1=$ high $)$.

Date and cause of death was cross-checked with that data held by the National Cancer Registry and the Registrar General (Scotland), and 1 December 2011 served as the censor date. Cancer-specific and overall survival were calculated from the date of post-operative blood sample until the date of death.

The study was approved by the Research Ethics Committee, Glasgow Royal Infirmary.

Statistics. The prognostic value of the pre-operative measure of the systemic inflammatory response was assessed using survival calculated from the date of the pre-operative blood test until the date of death. While the prognostic value of the post-operative measure of the systemic inflammatory response was assessed using survival calculated from the date of the post-operative blood test until the date of death.

Univariate survival analysis was carried out using the KaplanMeier method with the log rank test and associations between categorical variables examined using the $\chi^{2}$-test for linear trend. The Wilcoxon Rank test was used to test the related samples preoperative mGPS and post-operative mGPS, and pre-operative NLR and post-operative NLR. Cox proportional hazard regression was used to calculate hazard ratio (HR) and 95\% confidence intervals (95\% CI). A $P$-value of $<0.05$ was considered to be significant. Analysis was performed using SPSS software version 19 (SPSS Inc., Chicago, IL, USA).

\section{RESULTS}

Three hundred and twenty-six patients undergoing potentially curative resection for CRC were identified from a prospectively maintained database and studied. Full biochemical and haematological data both pre- and post-operatively were available for 206 patients. The patient characteristics of those patients with longitudinal measurements were not significantly different to those without (data not shown).

The baseline characteristics of patients for whom full pre- and post-operative biochemical and haematological data were available are shown in Table 1 . One hundred and twenty-nine (63\%) patients underwent surgery for colonic tumours, whereas 77 (37\%) had surgery for rectal tumours. The majority of patients were $>65$ years old (64\%), were male (58\%), had TNM stage I/II disease, and were carried out electively. Fifty-eight $(28 \%)$ patients received adjuvant therapy following resection of the primary tumour.

Twenty five patients received neoadjuvant therapy. Although pre- $(P<0.005)$ and post-operative $(P<0.001)$ NLR were associated with administration of neoadjuvant therapy, there was no association between pre- or post-operative mGPS.

The majority of patients (91\%) included in the present study were elective resections.

Emergency presentation was significantly associated with a higher pre-operative mGPS $(P<0.001)$, whereas pre-operative NLR was not $(P=0.116)$. However, in the present, relatively small study, mode of presentation itself was not significantly associated with cancer-specific survival and was not significantly associated with either post-operative mGPS or NLR.

With regard to more traditional predictors of poor outcome, neither pre- or post-operative mGPS, or pre- or post-operative NLR were associated with TNM stage, whereas only pre-operative NLR $(P<0.05)$ and post-operative mGPS $(P<0.05)$ were associated with margin status.

In the 206 patients with longitudinal measurements, 74 (36\%) patients had an elevated mGPS, pre-operatively. Following resection, $66(32 \%)$ had an elevated mGPS. With regard to the NLR, 46 (22\%) had an elevated NLR pre-operatively, whereas 36 (18\%) had an elevated NLR post-operatively.

Analysis of the relationship between the pre- and post-operative samples using the Wilcoxon Rank test, showed no significant difference between both the pre- and post-operative mGPS, and the pre- and post-operative NLR (Table 1, both $P>0.10$ ).

Of the 132 patients with an mGPS of 0 pre-operatively, 98 (74\%) had an mGPS of 0 on follow-up, 9 (7\%) had an mGPS of 1 on follow-up, and 25 (19\%) had an mGPS of 2 on follow-up. Of the 33 patients with an mGPS of 1 pre-operatively, 20 (61\%) had an mGPS of 0 on follow-up, 3 (9\%) patients had an mGPS of 1 on follow-up, and $10(30 \%)$ had an mGPS of 2 on follow-up. Of the 41 
Table 1. Baseline clinicopathological characteristics of patients undergoing potentially curative resection for colorectal cancer

Number of patients $(\boldsymbol{n}=\mathbf{2 0 6})$

\begin{tabular}{|c|c|c|}
\hline \multicolumn{3}{|l|}{ Age } \\
\hline$<65 / 65-74 />75$ & \multicolumn{2}{|c|}{$74(36 \%) / 79(38 \%) / 53(26 \%)$} \\
\hline \multicolumn{3}{|l|}{ Sex } \\
\hline Male/female & \multicolumn{2}{|c|}{$120(58 \%) / 86(42 \%)$} \\
\hline \multicolumn{3}{|l|}{ Presentation } \\
\hline Elective/emergency & \multicolumn{2}{|c|}{$187(91 \%) / 19(9 \%)$} \\
\hline \multicolumn{3}{|l|}{ Site } \\
\hline Colon/rectum & \multicolumn{2}{|c|}{$129(63 \%) 77(37 \%)$} \\
\hline T-stage & Colon & Rectum \\
\hline T1 & $8(4 \%)$ & $6(3 \%)$ \\
\hline T2 & $12(6 \%)$ & $9(4 \%)$ \\
\hline T3 & 63 (31\%) & 49 (24\%) \\
\hline T4 & $46(22 \%)$ & $12(6 \%)$ \\
\hline \multicolumn{3}{|l|}{ TNM stage } \\
\hline I & \multicolumn{2}{|c|}{$32(16 \%)$} \\
\hline ॥ & \multicolumn{2}{|c|}{85 (41\%) } \\
\hline III & \multicolumn{2}{|c|}{87 (42\%) } \\
\hline IV & \multicolumn{2}{|c|}{$2(1 \%)$} \\
\hline
\end{tabular}

\section{Adjuvant chemotherapy}

\begin{tabular}{|c|c|c|c|}
\hline \multirow{2}{*}{$\begin{array}{l}\text { No/yes } \\
\text { Measurement of } \\
\text { systemic } \\
\text { inflammatory } \\
\text { response (TNM I-IV) }\end{array}$} & \multicolumn{3}{|c|}{$148(72 \%) / 58(28 \%)$} \\
\hline & $\begin{array}{c}\text { Pre- } \\
\text { operative } \\
(n=206)\end{array}$ & $\begin{array}{c}\text { Post- } \\
\text { operative } \\
(n=206)\end{array}$ & $\boldsymbol{P}$-value* \\
\hline \multicolumn{4}{|l|}{ mGPS } \\
\hline $\begin{array}{l}0 \\
1 \\
2\end{array}$ & $\begin{array}{r}132(64 \%) \\
33(16 \%) \\
41(20 \%)\end{array}$ & $\begin{array}{c}140(68 \%) \\
18(9 \%) \\
48(23 \%)\end{array}$ & 0.926 \\
\hline \multicolumn{4}{|l|}{ NLR } \\
\hline $\begin{array}{l}0(<5) \\
1(>5)\end{array}$ & $\begin{array}{r}161(78 \%) \\
45(22 \%) \\
\end{array}$ & $\begin{array}{r}170(82 \%) \\
36(18 \%) \\
\end{array}$ & 0.216 \\
\hline $\begin{array}{l}\text { Measurement of } \\
\text { systemic } \\
\text { inflammatory } \\
\text { response (TNM I-II) }\end{array}$ & $\begin{array}{c}\text { Pre- } \\
\text { operative } \\
(n=117)\end{array}$ & $\begin{array}{c}\text { Post- } \\
\text { operative } \\
(n=117)\end{array}$ & $P$-value* \\
\hline \multicolumn{4}{|l|}{ mGPS } \\
\hline $\begin{array}{l}0 \\
1 \\
2\end{array}$ & $\begin{array}{l}74(63 \%) \\
17(15 \%) \\
26(22 \%)\end{array}$ & $\begin{array}{l}82(70 \%) \\
10(9 \%) \\
25(21 \%)\end{array}$ & 0.427 \\
\hline \multicolumn{4}{|l|}{ NLR } \\
\hline $\begin{array}{l}0(<5) \\
1(>5)\end{array}$ & $\begin{array}{l}90 \text { (77\%) } \\
27(23 \%) \\
\end{array}$ & $\begin{array}{l}93(79 \%) \\
24(21 \%) \\
\end{array}$ & 0.577 \\
\hline
\end{tabular}

Abbreviations: $m G P S=$ modified Glasgow Prognostic Score; NLR= neutrophil-lymphocyte ratio; $\mathrm{TNM}=$ tumour, node, metastasis. *Wilcoxon Rank test.

patients with an mGPS of 2 pre-operatively, 22 (54\%) had an mGPS of 0 on follow-up, 6 (15\%) had an mGPS of 1 on follow-up, and $13(32 \%)$ had an mGPS of 2 on follow-up. Of the 161 patients
Table 2. The relationship between clinicopathological characteristics and survival in patients undergoing potentially curative resection for

colorectal cancer

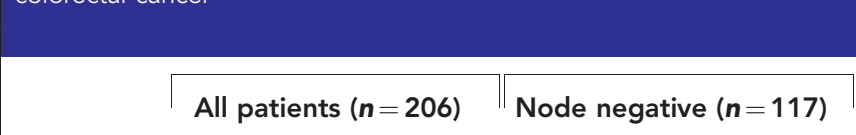

\section{Cancer-specific survival Cancer-specific survival Univariate analysis Univariate analysis}

\begin{tabular}{|l|c|c|c|c|c|c|}
\hline & $\mathrm{HR}$ & $95 \% \mathrm{Cl}$ & $P$-value & $\mathrm{HR}$ & $95 \% \mathrm{Cl}$ & P-value \\
\hline $\begin{array}{l}\text { Age (<65/ } \\
\text { 65-74/ } \\
>75)\end{array}$ & 0.79 & $0.49-1.27$ & 0.330 & 0.10 & $0.24-1.14$ & 0.101 \\
\hline $\begin{array}{l}\text { Gender } \\
\text { (male/ } \\
\text { female) }\end{array}$ & 1.24 & $0.58-2.63$ & 0.570 & 4.23 & $0.92-19.41$ & 0.064 \\
\hline $\begin{array}{l}\text { Site (colon/ } \\
\text { rectum) }\end{array}$ & 1.03 & $0.49-2.19$ & 0.930 & 1.55 & $0.49-4.80$ & 0.450 \\
\hline $\begin{array}{l}\text { T-stage } \\
\text { (T1/T2/T3/ } \\
\text { T4) }\end{array}$ & 2.75 & $0.49-5.07$ & 0.001 & 1.69 & $0.77-3.70$ & 0.190 \\
\hline $\begin{array}{l}\text { TNM stage } \\
\text { I/II/II/IV }\end{array}$ & 2.31 & $1.28-4.17$ & 0.005 & 4.13 & $0.53-32.08$ & 0.180 \\
\hline $\begin{array}{l}\text { Pre- } \\
\text { operative } \\
\text { mGPS (0/ } \\
1 / 2)\end{array}$ & 1.94 & $1.17-3.21$ & 0.010 & 2.57 & $1.10-5.96$ & 0.028 \\
\hline $\begin{array}{l}\text { Pre- } \\
\text { operative } \\
\text { NLR (<5/ } \\
>5 \text { ) }\end{array}$ & 3.28 & $1.36-7.93$ & 0.008 & 2.08 & $0.62-2.09$ & 0.233 \\
\hline $\begin{array}{l}\text { Post- } \\
\text { operative } \\
\text { mGPS (0/ } \\
1 / 2)\end{array}$ & 3.31 & $2.15-5.09$ & $<0.001$ & 4.81 & $2.13-10.83$ & $<0.001$ \\
\hline $\begin{array}{l}\text { Post- } \\
\text { operative } \\
\text { NLR (<5/ } \\
>5 \text { ) }\end{array}$ & 3.07 & $1.42-6.62$ & 0.004 & 3.10 & $0.98-9.84$ & 0.054 \\
\hline Abbreviations: Cl=confidence intervals; HR=hazard ratio. & & & & \\
\hline
\end{tabular}

with an NLR of 0 pre-operatively, 139 (86\%) had an NLR of 0 on follow-up, whereas 22 (14\%) patients had an NLR of 1 on followup. Of the 45 patients with an NLR of 1 pre-operatively, 31 (69\%) patients had an NLR of 0 on follow-up, whereas 14 (31\%) had an NLR of 1 on follow-up. Similarly, there was no significant difference between both the pre- and post-operative mGPS and the pre- and post-operative NLR in patients with node-negative disease (both $P>0.10$ ).

The relationship between clinicopathological characteristics and survival in patients undergoing potentially curative resection for CRC are shown in Table 2. In the present study, the minimum follow-up period was 12 months and the median follow-up period of those who remained alive was 36 months (range 12-71) months. On follow-up, 41 patients died, of which 29 patients died of CRC and 12 from other causes. No patients were lost to follow-up.

On univariate survival analysis in 206 patients, T-stage $(P<0.001), \quad$ TNM stage $(P=0.005), \quad$ pre-operative mGPS $(P<0.05$, Figure 1a) and NLR $(P<0.05$, Figure 1b) were significantly associated with cancer-specific survival. 
A

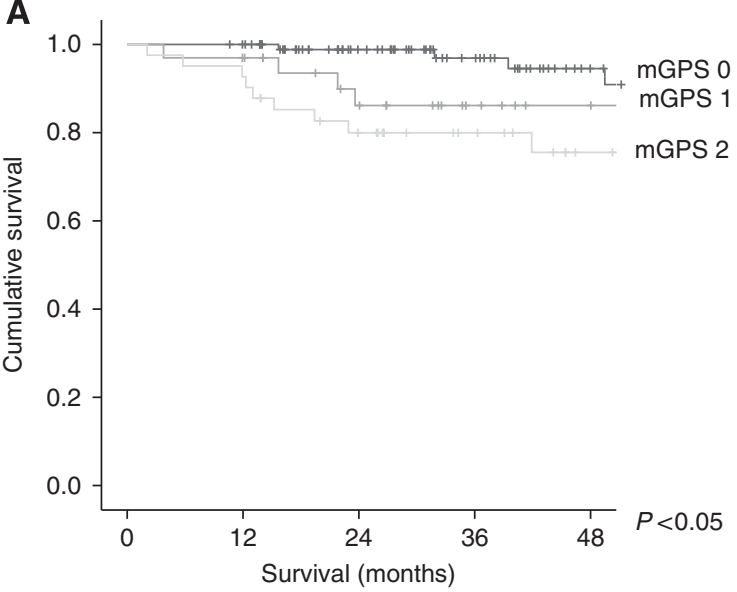

\begin{tabular}{|l|c|c|c|c|c|}
\hline No. at risk & \multicolumn{7}{|l|}{} \\
\hline & 0 & 12 & 24 & 36 & 48 \\
\hline mGPS 0 & 132 & 125 & 86 & 54 & 32 \\
\hline mGPS 1 & 33 & 32 & 23 & 15 & 11 \\
\hline mGPS 2 & 41 & 38 & 29 & 21 & 13 \\
\hline
\end{tabular}

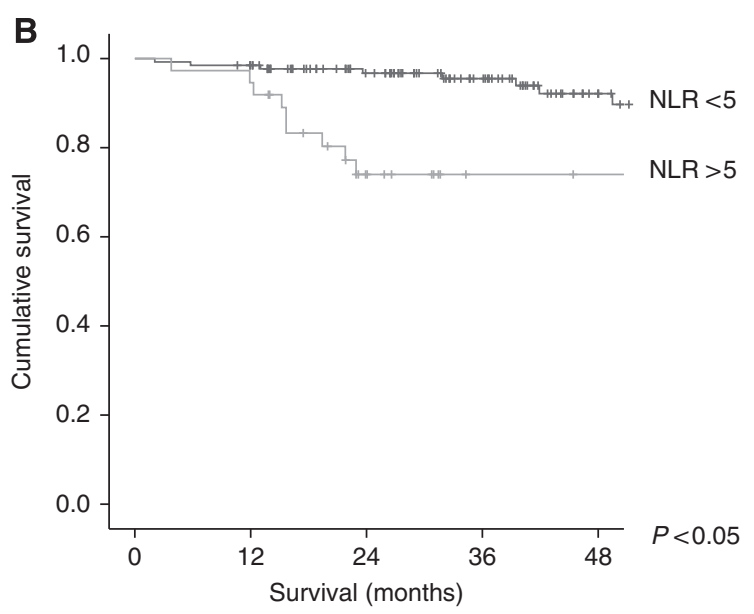

\begin{tabular}{|l|c|c|c|c|c|}
\cline { 1 - 5 } No. at risk & \multicolumn{7}{|l|}{} \\
\cline { 1 - 5 } & 0 & 12 & 24 & 36 & 48 \\
\hline NLR $<5$ & 161 & 151 & 111 & 75 & 43 \\
\hline NLR $>5$ & 45 & 44 & 27 & 15 & 13 \\
\hline
\end{tabular}

Figure 1. The relationship between pre-operative assessment of systemic inflammation as evidence by mGPS (A) and NLR (B) and cancerspecific survival in patients undergoing potentially curative resection of CRC.
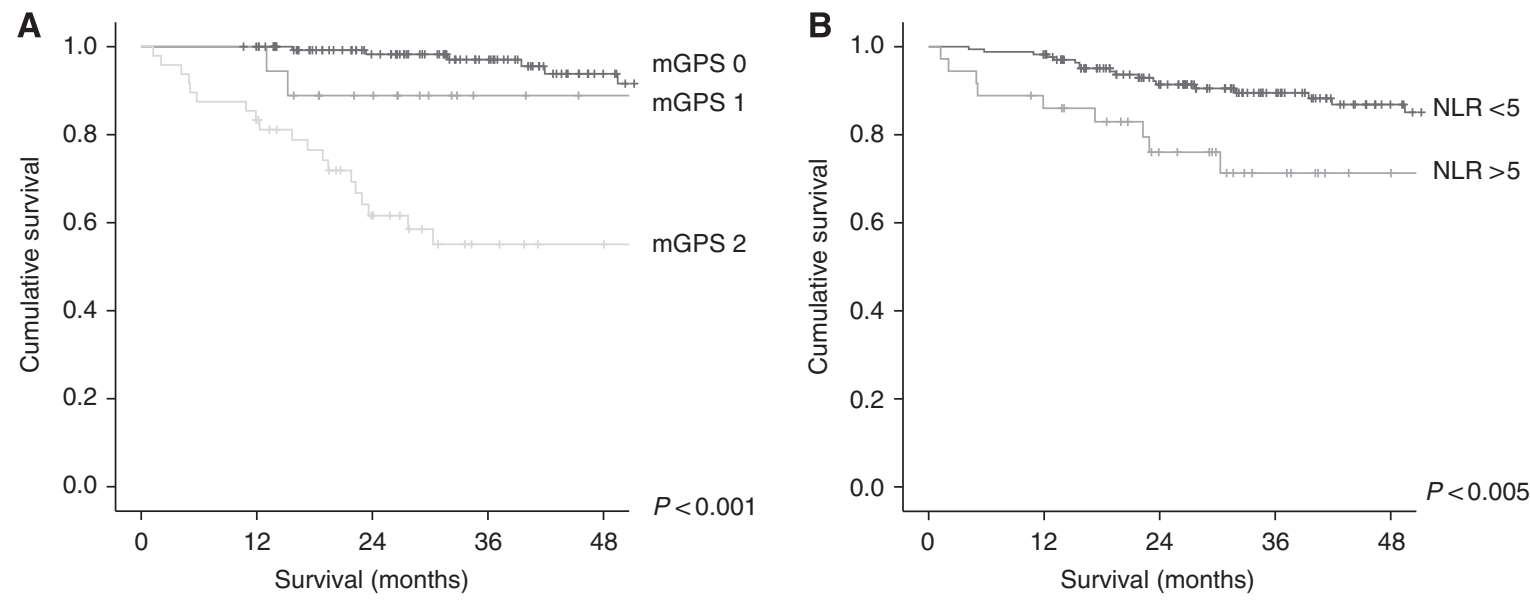

\begin{tabular}{|l|c|c|c|c|c|}
\cline { 1 - 5 } No. at risk & \multicolumn{1}{l|}{} \\
\cline { 1 - 5 } & 0 & 12 & 24 & 36 & 48 \\
\hline mGPS 0 & 140 & 120 & 90 & 59 & 39 \\
\hline mGPS 1 & 18 & 15 & 10 & 3 & 2 \\
\hline mGPS 2 & 48 & 32 & 20 & 12 & 9 \\
\hline
\end{tabular}

\begin{tabular}{|l|c|c|c|c|c|}
\hline \multicolumn{1}{|l|}{ No. at risk } & \multicolumn{1}{|l|}{} \\
\hline & 0 & 12 & 24 & 36 & 48 \\
\hline NLR $<5$ & 170 & 141 & 102 & 65 & 46 \\
\hline NLR $>5$ & 36 & 26 & 18 & 9 & 4 \\
\hline
\end{tabular}

Figure 2. The relationship between post-operative assessment of systemic inflammation as evidence by mGPS (A) and NLR (B) and cancerspecific survival in patients undergoing potentially curative resection of CRC.

Similarly, on univariate survival analysis post-operative mGPS $(P<0.001$, Figure 2a) and NLR $(P<0.005$, Figure $2 \mathrm{~b})$ were both significantly associated with cancer-specific survival.

On multivariate survival analysis, comparing pre-operative mGPS with NLR, both pre-operative mGPS (HR 1.97, confidence intervals $(\mathrm{CI}) 1.16-3.34, P<0.05)$ and NLR (HR 3.07, CI 1.23-7.63, $P<0.05)$ were independently associated with reduced cancerspecific survival (mGPS and NLR). When the same multivariate comparison was carried out on post-operative measurements, only the post-operative mGPS was independently associated with the cancer-specific survival (HR 4.81, CI 2.13-10.83, $P<0.001$ ).

On univariate survival analysis in 117 node-negative patients, only pre-operative mGPS $(P<0.05)$ was associated with cancerspecific survival. On univariate analysis using the date of post- operative sample to the date of death, only post-operative mGPS $(P<0.001)$ was significantly associated with cancer-specific survival.

\section{DISCUSSION}

In the present study, we report, for the first time, a longitudinal comparison of systemic inflammation-based prognostic scores in patients with CRC. The results are consistent with previous studies that have reported the prognostic value of the pre-operative assessment of the mGPS and the NLR. Further, the post-operative assessments (3-6 months) also had prognostic value in patients 
with CRC. Therefore, it is clear that the persistent elevation of the systemic inflammatory response, before and following resection of $\mathrm{CRC}$, is associated with poor survival. Taken together, there is clearly a role for the routine monitoring of the systemic inflammatory response in patients undergoing potentially curative resection of CRC.

In the present study, it was of interest that the systemic inflammatory response, whether measured using the mGPS or NLR, was not subject to significant overall change from the pre- to post-operative period. The majority of patients, before surgery, had no evidence of a systemic inflammatory response. In these patients, the majority remained systemically noninflamed following surgery. Of the patients who had evidence of a systemic inflammatory response before surgery, a small proportion became systemically noninflamed following resection of the primary tumour. The consequence of these changes may be that the post-operative measurement of the systemic inflammatory response better predicts outcome (McMillan et al, 2003; Jamieson et al, 2005). Indeed, these results are consistent with previous studies that reported the prognostic value of CRP alone, both pre- and postoperatively (McMillan et al, 2003; Jamieson et al, 2005). When the post-operative mGPS and NLR were compared, it was clear that the mGPS had superior prognostic value. Further studies are warranted.

Recently, it has been reported that the NLR was associated with a higher mortality in patients with coronary artery disease (Park et al, 2013). Given that this is a major cause of non-cancer death, it is probable that some of the patients in the present study died of coronary artery disease. However, the small numbers of non-cancer deaths in the present study precluded meaningful statistical analysis.

In the present study, we assessed the prognostic value of longitudinal measurements of systemic inflammation in patients undergoing curative resection of the CRC. In our centre, CEA is not routinely analysed because of the well-established findings that pre-operative CEA poorly predicts outcome in patients undergoing curative resection of CRC (Lewi et al, 1984).

Although there is good evidence that presence of the systemic inflammatory response, evidenced by mGPS or NLR, is associated with poorer outcomes in patients with cancer (McMillan, 2012), (Guthrie et al, 2013) the mechanism underlying persistent activation of the systemic inflammatory response in patients who have undergone resection of the primary CRC remains unclear. It may be secondary to chronic dysregulation of immune and inflammatory responses owing to activation by micrometastatic disease or non-malignant disease invoking tissue injury/necrosis. Plausible mediators of these immune and inflammatory responses are the pro-inflammatory cytokines that have been reported to be present at elevated concentrations in the circulation of patients with CRC (Kantola et al, 2012; Guthrie and McMillan, 2013). In particular, the pleiotropic pro-inflammatory cytokine interleukin-6 (IL-6), upregulated by tissue injury/necrosis and inflammatory cells such as macrophages, has the ability to activate and maintain the systemic inflammatory response through trans-signalling pathways involving the soluble IL-6 receptor (Guthrie et al, 2012).

The reasons for those patients with persistently activated systemic inflammatory responses having worse prognosis is becoming increasingly clear. In addition to the recognised detrimental effect on nutritional and functional status (McMillan, 2012), there is increasing evidence that an elevated systemic inflammatory response is associated with dysregulated, in particular upregulation of innate immune responses and downregulation of adaptive immune responses promoting tumour dissemination and progression (Roxburgh and McMillan, 2010; Guthrie et al, 2012; McMillan, 2012).

In summary, the results of the present study support the longitudinal assessment of the systemic inflammatory response, in particular using the mGPS, in patients undergoing potentially curative resection for CRC.

\section{REFERENCES}

AJCC (2010) Colon and Rectum Cancer Staging. AJCC Cancer Staging Manual. 7th Edn. Springer, New York.

Cancerstats (2010) Cancer Research UK. Available at http://www. cancerresearchuk.org/cancer-info/cancerstats/types/bowel/?script=true.

Guthrie G, McMillan DC (2013) Reply:Comment on 'stage-dependent alterations of the serum cytokine pattern in colorectal carcinoma'. $\mathrm{Br} \mathrm{J}$ Cancer 108: 1915-1916.

Guthrie GJ, Roxburgh CS, Horgan PG, McMillan DC (2012) Does interleukin6 link explain the link between tumour necrosis, local and systemic inflammatory responses and outcome in patients with colorectal cancer? Cancer Treat Rev 39: 89-96.

Guthrie GJK, Charles KA, Roxburgh CS, Horgan PG, McMillan DC, Clarke SJ (2013) The systemic inflammation-based neutrophil-lymphocyte ration: experience in patients with cancer. Crit Rev Oncol Hematol S1040-8428: 00070-X.

Jamieson NB, Glen P, McMillan DC, Mckay CJ, Foulis AK, Carter R, Imrie CW (2005) Systemic inflammatory response predicts outcome in patients undergoing resection for ductal adenocarcinoma head of pancreas. $\mathrm{Br} \mathrm{J}$ Cancer 92: 21-23.

Kantola T, Klintrup K, Vayrynen JP, Vornanen J, Bloigu R, Karhu T, Herzig KH, Napankangas J, Makela J, Karttunen TJ, Tuomisto A, Makinen MJ (2012) Stage-dependent alterations of the serum cytokine pattern in colorectal carcinoma. Br J Cancer 107: 1729-1736.

Lewi H, Blumgart LH, Carter DC, Gillis CR, Hole D, Ratcliffe JG, Wood CB, Mcardle CS (1984) Pre-operative carcino-embryonic antigen and survival in patients with colorectal cancer. Br J Surg 71: 206-208.

McMillan DC (2012) The systemic inflammation-based Glasgow Prognostic Score: a decade of experience in patients with cancer. Cancer Treat Rev 39: 534-540.

McMillan DC, Canna K, Mcardle CS (2003) Systemic inflammatory response predicts survival following curative resection of colorectal cancer. Br J Surg 90: $215-219$.

Oliphant R, Nicholson G, Horgan P, Molloy R, McMillan D, Morrison D (2013) Deprivation and colorectal cancer surgery: longer-term survival inequalities are due to differential postoperative mortality between socioeconomic groups. Ann Surg Oncol 20: 2132-2139.

Park JJ, Jang H-J, Oh I-Y, Yoon C-H, Suh J-W, Cho Y-S, Youn T-J, Cho G-Y, Chae I-H, Choi D-J (2013) Prognostic value of neutrophil to lymphocyte ratio in patients presenting with st-elevation myocardial infarction undergoing primary percutaneous coronary intervention. Am J Cardiol 111: $636-642$.

Proctor MJ, Morrison DS, Talwar D, Balmer SM, Fletcher CD, O'reilly DS, Foulis AK, Horgan PG, McMillan DC (2011) A comparison of inflammation-based prognostic scores in patients with cancer. A Glasgow Inflammation Outcome Study. Eur J Cancer 47: 2633-2641.

Roxburgh C. S., McMillan D. C. (2010) Role of systemic inflammatory response in predicting survival in patients with primary operable cancer. Future Oncol 6: 149-163.

This work is published under the standard license to publish agreement. After 12 months the work will become freely available and the license terms will switch to a Creative Commons AttributionNonCommercial-Share Alike 3.0 Unported License. 